\title{
The Pump Condition Analysis in Variable Frequency Constant Pressure Water Supply System
}

\author{
Sun Xiao1,Wu Shang1, ${ }^{\mathrm{a}}$, Hu Weilin $1,{ }^{\mathrm{b}}$, Wu Yulong1 $1{ }^{\mathrm{c}}$ \\ Hunan University of Technology College of Mechanical Engineering, Zhuzhou 412007,China \\ agodlike_boss@163.com, ${ }^{\text {b}} 190709001 @ q q . c o m,{ }^{c} 88905149 @ q q . c o m$
}

\begin{abstract}
Key Words: Water Supply System Condition Analysis Energy Efficiency
Abstract: In view of the variable frequency constant pressure water supply system, according to the change of water consumption by users, analyzed the requests of pump number increase or decrease shall meeted,in guarantee of the premise of basic constant water pressure in pipe network, to make water pressure stable in pipeline, improve power saving rate, reduce system mechanical wear.
\end{abstract}

\section{Introduction:}

At present,based on the variable frequency speed regulation constant pressure water supply is the main way of urban water supply,this way can meet the demands of users,and it also can obtain economic benefits.In practice, Water supply system is often a few pumps operation in parallel.But in this way,there are some bad things, investment is very expensive, it is not clever to make all of the pumps can change the speed.So commonly used speed mixing water supply pumps and constant speed pumps. So,study the issue of water pumps switch has a certain practical significance.

When water pump unit switching, control the pumps increase or decrease based on:when the existing pumps of water supply pressure is insufficient,increase the number of pump; On the contrary,when the existing pumps of water supply pressure is too large,decrease the number of pump.

In variable frequency constant pressure water supply system add and subtract the situation of the pumps can be divided into the following two:

When existing have been working in the upper limit frequency variable frequency pump operation, and the actual pressure inside the pipe net value remains below the set pressure value, it is need to increasing the number of running pumps to improve pipe network water pressure to meet the requirements.

When existing have been working in the lower frequency variable frequency pump operation, and the actual pressure inside the pipe net value remains above the set pressure value, it is need to decreasing the number of running pumps to improve pipe network water pressure to meet the requirements.

In guarantee under the premise of basic constant water pressure in pipe network,pump unit under what conditions to switch or how to control the switch to complete,not only can make the switch of the unit too often, and can continue to provide stable and reliable water supply pressure?

\section{The basic characteristics of water supply system}

The principle of constant pressure water supply:

The principle of variable frequency speed regulation constant pressure water supply system: Through online pressure sensor will be installed in the outlet pipe export pressure into electrical 
signals transmitted into the controller, after comparing with the given pressure parameters, adjust the parameter, Input to the frequency converter, control of ac motor speed to control the output flow and pressure of the network, In the pipeline pressure value to set value.According to the variation of water users, continuously controlled by the controller work frequency converter speed regulating of water pump and water pump number increase or decrease,closed-loop control to achieve the constant pressure water supply.

The characteristics of the pump running:

When two or more than two pumps in series,flow has no big changes, but the lift superposition.Two or more than two of the operation of the pump to the same pressure pipeline fluid way called parallel operation.In parallel,lift has no big change,flow superposition. The purpose of parallel operation is to increase the flow of the fluid,suitable for big flow changes, using a large pump operation economical efficiency. At the same time, it can have a spare pump when the pump parallel operation,in order to ensure running safety and reliability of the system.

Frequency conversion water supply features:

Due to the constraints in the water supply system, the frequency regulating range is limited.It can't infinitely increase or decrease.Upper limit frequency:According to the different countries standard,the inverter frequency adjustment has a limit.The lower frequency: The lowest frequency of the inverter is $0 \mathrm{~Hz}$.But in the practical application, due to the change of network impedance,the requirement of pipe network system.The speed of the pump not unlimited access to reduce.That is to say, the output frequency of frequency converter has a lower value. Otherwise due to the pump efficiency is reduced,the actual pipe network pressure is not because of motor frequency falls.And there is no effective power output,instead increase the energy consumption. The lower frequency specific concrete numerical value with the pump characteristic and system used in the place.

\section{Variable frequency pump and Rated frequency pump diagram analysis of joint operation}

When variable frequency pump and rated frequency pump parallel running,the lift is equal.The Working point of water pump in parallel operation,is decided by total performance curve and total Pipeline characteristic curve.A single pump frequency conversion runtime, Water pump lift of water only when is greater than the net head,Therefore, the starting point of the pipe resistance curve shall be the net head height $H_{0}$.

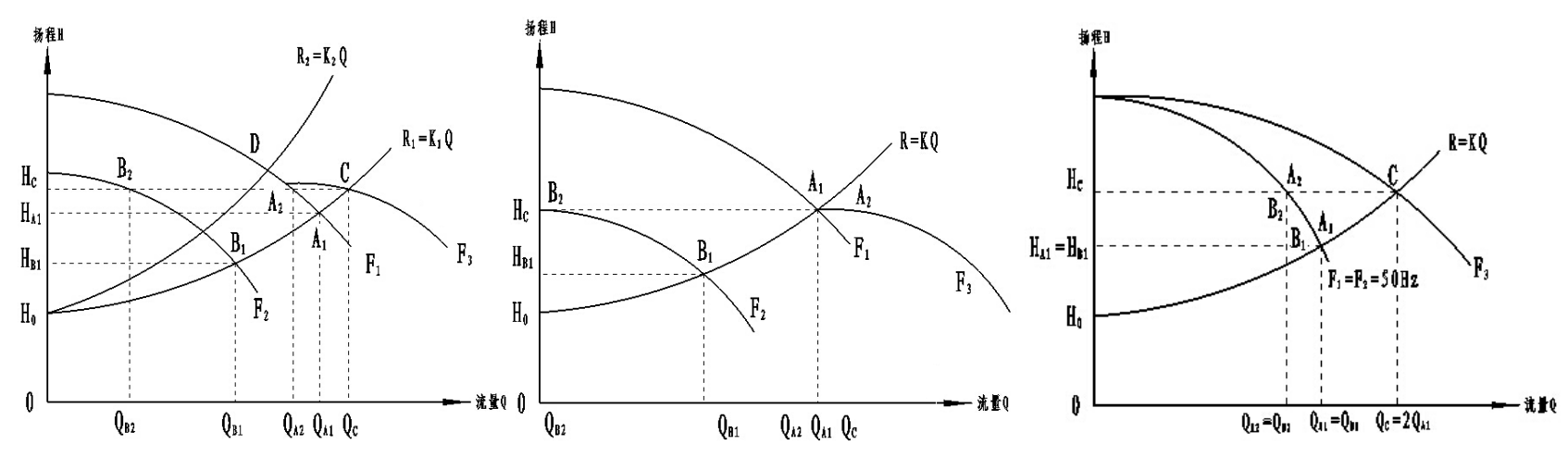

Fig.1 Variable frequency pump and rated frequency pump parallel runtime performance curve

Fig.2 The performance curve of variable frequency pump operate in the lowest frequency paralleled with rated frequency pump

Fig.3 The performance curve of variable frequency pump operate in the highest frequency paralleled with rated frequency pump

In figure $1, \mathrm{~F}_{1}$ is the performance curve of rated frequency pump separate runtime,working point is $A_{1} \cdot F_{2}$ is the performance curve of variable frequency pump in $F_{2}$ single runtime,working point is $\mathrm{B}_{1} \cdot \mathrm{F}_{3}$ is variable frequency pump and rated frequency pump parallel runtime performance 
curve,working point is $\mathrm{C}$.The figure shows,at working point $\mathrm{C}$, the lift and the flow are more than two pump separate values at runtime, at the sametime,variable frequency pump and rated frequency pump have the same lift number.

Figure2 and figure 3 each shows the performance curve of variable frequency pump operate in the lowest frequency paralleled with rated frequency pump and the performance curve of variable frequency pump operate in the highest frequency paralleled with rated frequency pump.

The lift of variable frequency pump can not more than the lift of rated frequency pump,the variable frequency pump's flow be close to 0.At this time, paralleled performance curve is coincide to the rated frequency pump single running, the variable frequency pump offer no flow but still take away energy.So, should avoid such a situation.

\section{Control process}

To ensure the pump run in efficient and safety environment and also can adjust the flow well,it can using "one variable frequency pump and few rated frequency pump" or "synchronous frequency conversion". Considering the control of the cost, this study adopt the former way.Figure 4 shows the principle diagram of the system.

Taking an example of three pumps:When a constant speed pump paralleled with a variable frequency pump,and pipe network pressure is less than the set pressure value:If the frequency of variable frequency pump not reached the maximum frequency, it should be improve the inverter output frequency; If the frequency of variable frequency pump have reached the maximum frequency,still not meet the set requirements, the controller should be start a new rated frequency pump, and control inverter output frequency from low to high, until meet the pressure requirements. On the contrary,when pipe network pressure is more than the set pressure value:If the frequency of variable frequency pump not reached the minimum frequency,it should be reduce the inverter output frequency; If the frequency of variable frequency pump have reached the miniimum frequency,still not meet the set requirements, the controller should be shut down a rated frequency pump, and control inverter output frequency from high to low, until meet the pressure requirements.

The controller through comparing the current pressure pressure and set pressure,decision it is to increase or reduce the speed of the motor.Through judge whether the frequency of the inverter get to the highest frequency or lowest frequency,decision it is to increase or reduce the number of the rated frequency pump.But only conditions meet the upper and lower limits, Controller to add and subtract the pump operation still exist certain problems.Add add the following two switching conditions:

Margin conditions: If the output of the converter has reached its maximum frequency,only when the set pressure value is higher than the actual water supply pressure $\Delta P$ is allowed to increase the pump ;If the output of the inverter for the lower frequency,only when the set switch pressure is less than the actual pressure of water supply $\Delta P$ is allowed to increase the pump .The addition of the condition is to ensure that the actual water pressure have a certain distance from ideal water supply pressure,won't appear the phenomenon that after adding pump the pressure get too large immediately.

Time condition: On the basis of the margin conditions, when the current work condition inverter output to the maximum (minimum) frequency,the actual pipe network pressure is stilllessthan(greater than) the set pressure value, in this state delay work $1 \mathrm{~min}$.If the actual water pressure is still meet margin conditions, the controller to perform add(reduce) pump operation.Otherwise, maintain the current working status. The addition of the condition is to avoid the pressure measurements have peak pressure and in a short period of time pressure get too 
large.Figure 5 shows the system operation flow.

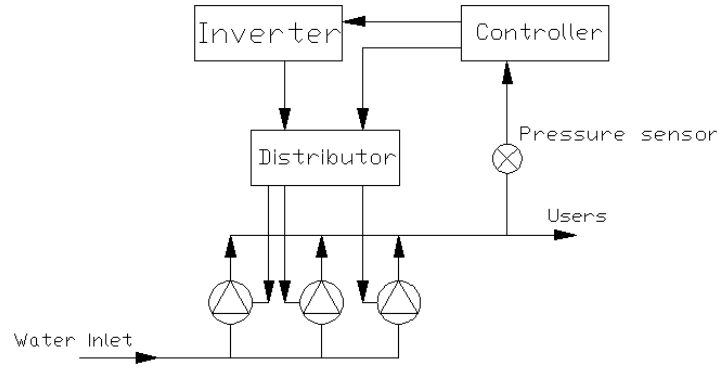

Fig.4 The principle diagram of the system

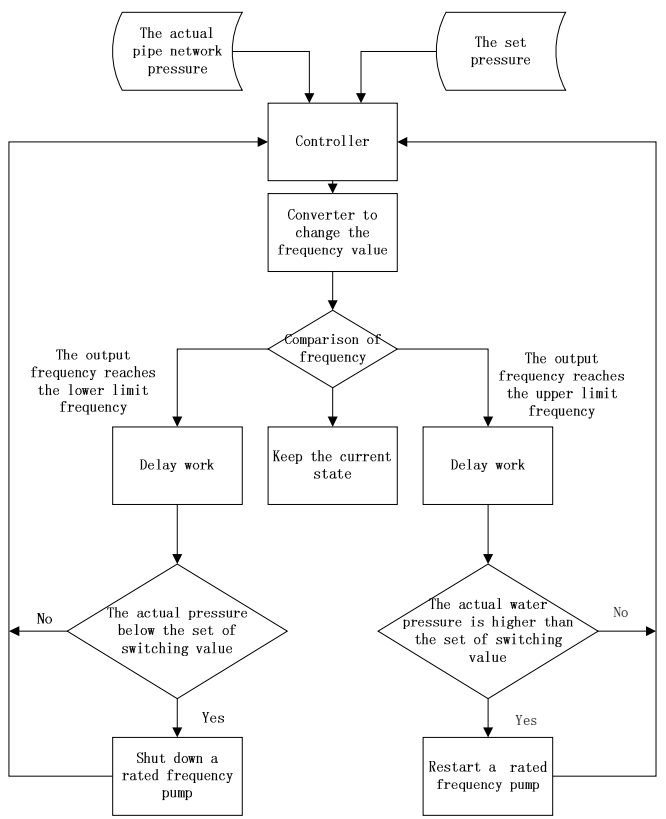

Fig.5 System operation flow chart

\section{References}

[1] Mollov S,Babuska R, Abonyi J,Verbruggen H B.Effective optimization for fuzzy model predictive control . IEEE Transactions on Fuzzy Systems, 2004 ,12(5).

[2] Takagi T,Sugeno M.Fuzzy identification of systems and its applications to modeling and control .IEEE Transactions on Systems,Man, and Cybernetics,1985,15:116-132.

[3] Tanaka K,Sugeno M.Stability analysis and design of fuzzy control systems.Fuzzy Sets and Systems, 1992,45:135-156.

[4] The Low Carbon Technology for Frequency Constant Pressure Water Supply Control and Application.Journal of Hunan University of Technology,2014,28:98-103.(In Chinese)

[5] Maeda M.Murskami S. Self-Tuning fuzzy controller. Fuzzy Sets and Systems. 1992. 51:29-40.

[6] Vidyasaga. Control System Synthesis: A Fartorization Approach.Cambridge, MA:MIT Press 1985, 124 .

[7] S.-W.Kim and J.-J.Lee. Design of a Fuzzy Controller with Fuzzy Sliding Surface. Fuzzy Sets Syst., vol. 71, pp. $359-367,1995$.

[8] Chen G R. Conventional and Fuzzy PID Controllers: An Overview. Int J Intelligent Control and Systems, 2001,1(2):235-246.

[9] Bryan Coulbeck,Pressure Control of a Moscow Water Supply System Using Expert System Technology,Conference on Decision and Control Proceedings of the 35th,1996:4498-4499 . 